\title{
Epidemiological association of Campylobacter jejuni groups with pathogenicity-associated genetic markers
}

\author{
Andreas E Zautner ${ }^{*}$, Carolin Ohk, Abdul Malik Tareen, Raimond Lugert and Uwe Groß
}

\begin{abstract}
Background: Campylobacter jejuni, the most leading cause for bacterial gastroenteritis worldwide, shows a high genetic diversity among its isolates. Recently, we demonstrated the existence of six $C$. jejuni-groups by combining MLST with six genetic markers. These groups were further characterized by the detection of cj1321-cj1326, fucP, cj0178, cj0755/cfrA, ceuE, pldA, cstll, and cstIII in order (I.) to show further associations between these different genetic markers and MLST CCs. Moreover, different studies were able to associate several of these markers: a sialylated lipoologosaccharide $\left(\mathrm{cs} t / / / I I^{+}\right)$, the gamma-glytamyl-transpeptidase $\left(g g t^{+}\right)$, and the absence of a certain allele of the enterochelin-uptake-binding-protein $\left(c e u E_{11168}\right)$ with severe campylobacteriosis, bloody diarrhea and unpleasant outcome. Additionally more than half of human Campylobacter-isolates were assigned to a non-livestock clade associated with the absence of cj1321-cj1326. These isolates were considered as mere colonizers.

From the combination of marker genes, the ratio of human isolates in a specific group, and clinical data (II.) it should be demonstrated to which of the previous defined groups these Campylobacter-subpopulations, associated with higher virulence, correspond.

Results: Besides the marker gene pldA, all new estimated genetic markers show significant differences in their distribution among the various MLST-based groups. Especially the genes for cj1321-cj1326, fucP, cj0178, cj0755/cfrA are widely associated with each other and split the study population into two major and seven intermediate groups substantiating the previous group-definition, whereas cstll and cstIII indicate at least three groups following an independent distribution pattern.

Conclusions: Based on these data a group of $C$. jejuni-isolates characterized by the presence of ansB, dms $A$, ggt, and the absence of cj1365c, cj1585c, cj1321-cj1326, fucP, cj0178, cj0755/cfrA, and cstI/IIII was associated with a higher prevalence in human campylobacteriosis, bloody diarrhea as well as hospitalization and bears obviously a higher virulence for humans. In contrast to that better livestock-adapted groups characterized by the ability to utilize L-fucose and the presence of all of the five identified putative C. jejuni iron-uptake systems as well as cj1321-cj1326, cj1365c, cj1585c, and cstll and/or cstIII (sialylated lipoologosaccharide) is more prevalent in animal hosts and was secondary associated with less severe campylobacteriosis.
\end{abstract}

Keywords: Campylobacter jejuni, Subgroups, fucP, cj0178, cj0755/cfrA, LOS class, Flagellin glycosylation, Virulence

\footnotetext{
* Correspondence: azautne@gwdg.de

Universitätsmedizin Göttingen, Abteilung für Medizinische Mikrobiologie, Kreuzbergring 57, D-37075 Göttingen, Germany
} 


\section{Background}

The Gram-negative Epsilonproteobacterium Campylobacter jejuni, which is due to recent epidemiological data the most leading cause for bacterial gastroenteritis and Guillain-Barré-syndrome (GBS) worldwide, shows a high genetic diversity among its isolates [1]. As consequence of this genetic and phenotypic diversity several C. jejuni subpopulations could be identified on the basis of the presence of non-ubiquitous genes [2]. In a previous study we could identify six $C$. jejuni groups combining multilocus sequence typing (MLST) with six genetic markers: ans B, dms $A$, ggt, cj1585c, cj1365c and dimeric tlp7 (Tlp7 $\mathrm{m}+\mathrm{Tlp} 7 \mathrm{c}$ ) [2]. Here we could in particular demonstrate that the genes ans $B$, $d m s A$, ggt occur together in a specific cj1585c- and cj1365c-negative isolate group [2].

Several studies were able to correlate further genetic markers with clinical parameters. Thus, the question was addressed how a sialylated lipoologosaccharide (LOS) affects the severity of the Campylobacter-trigged diarrhea [3-5]. It was demonstrated that a sialylated LOS of the Campylobacter cell wall is associated with an increased occurrence of bloody diarrhea and a longer duration of symptoms [3-5].

Champion and coworkers made a further interesting finding. They demonstrated that $55.7 \%$ of $C$. jejuni isolates from human faeces belong to a non-livestock clade that misses the flagellin $O$-glycosylation cluster encoded by the genes cj1321-cj1326 [6]. Cj1321-cj1326-negative strains originate mostly from asymptomatic carriers and the environment. Thus, flagellin O-glycosylation may play as well a role in cell invasion, and in consequence for the virulence in humans.

Another study of Feodoroff and coworkers identified a C. jejuni-subpopulation in which they were able to detect the gamma-glytamyl-transpeptidase gene ( $g g t)$ but not the fucose permease gene $(f u c P)$, the phospholipase A gene (pldA) and the enterochelin-uptake-binding-protein gene (ceuE) using pldA- and ceuE-primers derived from the NCTC 11168 genome sequence (The corresponding genes are designated in the following as $p l d A_{11168}$ and $\left.c e u E_{11168}\right)$ [7]. These isolates could be associated with a higher rate of hospitalizations and bloody diarrhea [7].

To determine the distribution of these further genetic markers as well as their association with $a n s B, d m s A$, ggt, $c j 1585 c, c j 1365 c$ and dimeric tlp 7 and secondary their correlation with the clinical data of the above mentioned studies, we further characterized the same 266 isolates by screening for the presence of eight additional genetic markers: the flagellin $O$-glycosylation locus cj1321-cj1326 [6], the L-fucose permease gene fucP [8], the outer membrane siderophore receptor cj0178 $[9,10]$, the iron uptake protein/ferric receptor cj0755/cfrA $[9,10]$, the enterochelin uptake binding protein ceuE [11], the outer membrane phospholipase A pldA [12], as well as the lipooligosaccharide sialyltransferases $c s t I I$ and cstIII $[13,14]$.

\section{Results}

The frequency of all eight new determined genetic markers in all tested 266 isolates and in each subgroup is listed in Table 1. Additionally the ratio of human isolates as parameter for the clinical relevance of the particular isolate group is listed there. A pictorial representation of the marker gene distribution among the various subgroups as well as their isolate origin is shown in Figure 1.

The flagellin O-glycosylation locus cj1321-cj1326 as marker for livestock-associated strains could be detected in the majority of the isolate groups: $1 \mathrm{~A}, 1 \mathrm{~B}^{*}, 1 \mathrm{~B}^{* *}, 3 \mathrm{~A}$ and 4 , assuming their livestock association. In contrast to that, especially the groups $2 \mathrm{~A}+\mathrm{B}$ as well as $1 \mathrm{~B}^{* * * *}, 3 \mathrm{~B}$ and 5 were negative for this marker gene.

A comparable distribution pattern could be demonstrated for the fucP gene. The isolate groups $1 \mathrm{~A}, 1 \mathrm{~B}^{*}$, $1 \mathrm{~B}^{* *}, 3 \mathrm{~A}^{*}$ and 6 , are positive for this marker gene, whereas the $f u c P$ genes was nearly absent in the groups $1 \mathrm{~B}^{* * *}, 2 \mathrm{~A}+\mathrm{B}, 3 \mathrm{~A}^{* *}+\mathrm{B}$ and 4 .

Feodoroff and coworkers identified a subpopulation in which they were not able to detect ceuE using ceuEprimers derived from the NCTC 11168 genome sequence [7]. The same phenomenon was described by them for pldA using NCTC 11168 genome based primers, but here the differences were not significant [7]. Using additional forward primers based on the gnome sequence of the 81176 strain (see Table 2), we could detect ceuE and pldA in the whole test population. Using exclusively the NCTC 11168 genome based primers a significant lowered ceuEdetection rate was only observerd for group 2 isolates (24.0\%, $\mathrm{p}<0.002)$. There were no significant differences in the pldA detection using additional 81-176 genome-based primes in our study population.

Furthermore, we included the genes cj0178, an outer membrane siderophore receptor, and cj0755, an iron uptake protein (ferric receptor), in the panel of marker genes. The gene products of cj0178 and cj0755 are like enterochelin, CeuE, involved in the microbial iron uptake. Thus, it was, because of their functional association to CeuE, suggestible that they may be associated with bloody diarrhea like ceuE [7] as well. Both genes could be detected, mostly associated with each other, in more than $76 \%$ of all isolates. In the groups $2(\mathrm{~A}+\mathrm{B})$ and 4 they are nearly completely absent, whereas about $100 \%$ of the remaining groups are positive for both genes.

Additionally, we looked for the presence of cstII and cstIII in order to distinguish isolates with sialylated LOS from isolates with non-sialylated LOS. There are already more detailed studies associating MLST CC with certain LCC $[3,15,16]$ allowing us to associate a particular isolate group with specific LCC only on the basis of the 
Table 1 Distribution and association of genetic markers, LLC and MLST-CC within the determined subgroups

\begin{tabular}{|c|c|c|c|c|c|c|c|c|c|c|}
\hline \multirow[t]{2}{*}{ (sub-) group } & \multicolumn{9}{|c|}{ No. of isolates with marker gene/total no. (\%) } & \multirow[t]{2}{*}{ human origin } \\
\hline & cj1321-1326 & fucP & cj0178 & cj0755 & ceuE $E_{11168}^{1}$ & $p / d A_{11168}^{2}$ & cstll & cstIII & $\mathrm{LLC}^{3}$ & \\
\hline $1 \mathrm{a}$ & $38 / 38^{\#}(100)$ & $38 / 38^{\#}(100)$ & $38 / 38^{\#}(100)$ & $38 / 38^{\#}(100)$ & $38 / 38^{\#}(100)$ & $38 / 38^{\#}(100)$ & $13 / 38^{\circ}(34.2)$ & $33 / 38^{\#}(86.4)$ & C/A & $16 / 38(42.1)$ \\
\hline $1 b^{*}$ & $43 / 44^{\#}(97.7)$ & $44 / 44^{\#}(100)$ & $44 / 44^{\#}(100)$ & $44 / 44^{\#}(100)$ & $42 / 44^{\circ}(95.5)$ & $41 / 44(93.2)$ & $16 / 44^{\circ}(36.4)$ & $37 / 44^{\#}(84.1)$ & $C / A / B$ & 19/44(43.2) \\
\hline $1 b^{* *}$ & $38 / 38^{\#}(100)$ & $36 / 38^{\#}(94.7)$ & $37 / 38^{\#}(97.4)$ & $38 / 38^{\#}(100)$ & 35/38(92.1) & $37 / 38^{\circ}(97.4)$ & $37 / 38^{\#}(97.4)$ & $2 / 38^{\#}(5.3)$ & B2 & 19/38(50.0) \\
\hline $1 b^{* * *}$ & $7 / 15(46.7)$ & $5 / 15^{\circ}(33.3)$ & $15 / 15^{\#}(100)$ & $15 / 15^{\#}(100)$ & $14 / 15^{\#}(93.3)$ & $15 / 15^{\#}(100)$ & $6 / 1 z(40.0)$ & $0 / 15^{\#}(0.0)$ & $B, D$ & $9 / 15(60.0)$ \\
\hline $2 a$ & $2 / 17^{\#}(11.8)$ & $0 / 17^{\#}(0.0)$ & $0 / 17^{\#}(0.0)$ & $3 / 17^{\#}(0.0)$ & 12/17(70.6) & 14/17(82.4) & $16 / 17^{\#}(94.1)$ & $1 / 17^{\#}(5.9)$ & $\mathrm{A} 1 / \mathrm{B}$ & 8/17(47.1) \\
\hline $2 b$ & $3 / 34^{\#}(8.8)$ & $1 / 34^{\#}(2.9)$ & $1 / 34^{\#}(2.9)$ & $1 / 34^{\#}(2.9)$ & $26 / 34^{\circ}(76.5)$ & 29/34(85.3) & $5 / 34^{\#}(14.7)$ & $0 / 34^{\#}(0.0)$ & $\mathrm{D} / \mathrm{E} / \mathrm{H} / \mathrm{U}$ & $22 / 34^{\circ}(64.7)$ \\
\hline $3 a^{*}$ & $15 / 22(68.2)$ & $18 / 22^{\circ}(81.8)$ & $22 / 22^{\#}(100)$ & $22 / 22^{\#}(100)$ & 18/22(81.8) & 18/22(81.8) & $18 / 22^{\#}(81.8)$ & $1 / 22^{\#}(4.5)$ & - & $15 / 22^{\circ}(68.2)$ \\
\hline $3 a^{* *}$ & $16 / 19^{\circ}(84.2)$ & $2 / 19^{\#}(10.5)$ & $19 / 19^{\#}(100)$ & $19 / 19^{\#}(100)$ & $18 / 19^{\#}(94.7)$ & 11/19(57.9) & $12 / 19(63.2)$ & 7/19(36.8) & E & $4 / 19^{\circ}(21.1)$ \\
\hline $3 b$ & $2 / 11^{\circ}(18.2)$ & $0 / 11^{\#}(0.0)$ & $11 / 11^{\#}(100)$ & $11 / 11^{\#}(100)$ & $10 / 11(90.9)$ & $8 / 11(72.7)$ & $10 / 11(90.9)$ & 1/11(9.1) & - & 3/11(27.3) \\
\hline 4 & $3 / 8(37.5)$ & $0 / 8^{\#}(0.0)$ & $1 / 8^{\sharp}(12.5)$ & $0 / 8^{\#}(0.0)$ & $7 / 8(87.5)$ & $6 / 8(75.0)$ & $5 / 8(62.5)$ & $0 / 8^{\#}(0.0)$ & - & $2 / 8(25.0)$ \\
\hline 5 & $0 / 4^{\#}(0.0)$ & $1 / 4(25.0)$ & $4 / 4^{\#}(100)$ & $4 / 4^{\#}(100)$ & $4 / 4^{\#}(100)$ & $4 / 4^{\#}(100)$ & $2 / 4(50.0)$ & $0 / 4^{\#}(0.0)$ & - & $1 / 4(25.0)$ \\
\hline 6 & $3 / 9(33.3)$ & $9 / 9^{\#}(100)$ & $9 / 9^{\#}(100)$ & $9 / 9^{\#}(100)$ & $8 / 9(88.8)$ & 8/9(88.8) & $2 / 9^{\circ}(22.2)$ & $0 / 9^{\#}(0.0)$ & $A / D$ & $7 / 9(77.8)$ \\
\hline all & 170/266(63.9) & 154/266(57.9) & 204/266(76.7) & 208/266(78.2) & 232/266(87.2) & 229/266(86.1) & $142 / 266(53.4)$ & $82 / 266(30.8)$ & all & 128/266(48.1) \\
\hline
\end{tabular}

Marker genes cij1321-cj1326: O-linked flagellin glycosylation locus; fucP: L-fucose permease gene (cj0486); cj0178: outer membrane siderophore receptor; cj0755: iron uptake protein (ferric receptor cfrA); ceuE:

enterochelin uptake binding protein; pldA: outer membrane phospholipase A; cstll: LOS sialyltransferase II; cstlll: LOS sialyltransferase III; LLC: lipooligosaccharid (LOS) locus class; MLST-CC: Multilocus sequence typing clonal complex; footnotes: 'negative corresponds to only detectable using the forward primer based on the 81-176 genome sequence: $5^{5}$-GAT-AGA-GTC-GCA-GGC-GTT-CC-3"2 negative corresponds to only detectable using the forward primer based on the 81-176 genome sequence: 5'-AAA-CTT-ATG-CGT-TTT-T-3' ${ }^{3}$ LLC according to Habib et al. 2009 [3], Hotter et al. 2010 [15], Revez et al. 2011 [16]; p<0.05/” p<0.001 significance level in comparison to the remaining isolates belonging not to the corresponding group, additionally the values in subgroups with above average numbers of positive isolates are given in bold numbers; in the case of ceuE and pldA the NCTC 11168 typical allele presence is given in bold if the isolate numbers were above average. 
(See figure on previous page.)

Figure 1 MLST-sequence based UPGMA-tree and the arrangement of the six different marker genes within the six defined groups (twelve subgroups). On the left side the MLST-sequence based UPGMA-tree of 266 C. jejuni isolates is depicted. The numbers shown on the branches of the tree indicate the linkage distances. The right side of the table lists all isolates in the order of the UPGMA-tree depicting the source of the isolate, the presence or absence of the six marker genes and their belonging to one of the groups listed in Table 1. Source: Human isolates are marked blue, chicken isolates yellow, bovine isolates red, and turkey isolates green. Marker genes: Presence of a genetic marker is marked with a light red shade, absence with a light green shade. The marker genes from left to right are: cjj1321-6: 0-linked flagellin glycosylation locus; fucP: L-fucose permease gene (cj0486); cj0178: outer membrane siderophore receptor; cj0755: iron uptake protein (ferric receptor $(f r A)$; ceuE: enterochelin uptake binding protein; pldA: outer membrane phospholipase A; cstIl: LOS sialyltransferase II; cstIII: LOS sialyltransferase III; The last column gives the group according to Table 1: light grey $(1 \mathrm{~A})$, light yellow $\left(1 \mathrm{~B}^{*}\right)$ intense yellow $\left(1 \mathrm{~B}^{* *}\right)$, dark yellow $\left(1 \mathrm{~B}^{* * *}\right)$ cyan blue $(2 A)$, bondi blue $(2 B)$, carrot-orange $\left(3 A^{*}\right)$, orange-red $\left(3 A^{* *}\right)$; rust-red $(3 B)$, turquoise [4], red [5], steel-blue [6] and white (singeltons).

MLST-CC and the information about the absence or presence of cstII and cstIII (see Table 1 and Figure 1). Group $1 \mathrm{~A}$ and $1 \mathrm{~B} *$ were generally tested positive for cstIII. The subgroup named $1 \mathrm{~B}^{* *}$, which is comprised of CC 48 and CC 206 isolates, is only cstII but not cstIII positive. Isolates from the subgroup $1 \mathrm{~B}^{* * *}$ (CC 49 and CC 446) are partially positive, partially negative for cstII but generally cstIII-negative. All in all, 23 isolates are positive for cstII and cstIII. Most of these double-positive isolates belong to group 1 (87.0\%) and CC 21 (65.2\%).

The isolates of group $2 \mathrm{~A}$ are in the majority cstII-positive, in contrast to group $2 \mathrm{~B}$ isolates that are negative for both, cstII and cstIII, which means that these isolates bear a non-sialylated LOS. Most of the group 3 isolates are positive for cstII but not cstIII, besides a minority of CC 353 isolates that are cstIII-positive. The majority of isolates in the groups 4, 5, and 6 are cstII- and cstIIInegative (non-sialylated LOS).

Finally the ratio of human isolates in comparison to all animal isolates was significantly $(\mathrm{p}=0.04355)$ increased in the ggt-positive subgroup $2 \mathrm{~B}$, whereas the difference for the whole group $2(A+B)$ was increased but not significant. An increased ratio of human isolates could be also detected for the fucP-negative subpopulation $\left(\mathrm{p}\left(1 \mathrm{~B}^{* * *}+2\right)=0.04790\right)$ as well as the ceuE-negative (referring to a PCR using NCTC 11168-based primers) subpopulation $\left(\mathrm{p}\left(2+3 \mathrm{~A}^{*}\right)=0.00825\right)$. However, we could not detect any significant association between a particular animal host species and the presence of the eight tested genetic markers (results not shown). With the exception of group $1 \mathrm{~B}^{* *}$ with a significant $(\mathrm{p}=0.01374)$ lower hospitalization rate and group $3 \mathrm{~A}^{*}$ with a significant $(p=0.00020)$ lower rate of bloody diarrhea no significant differences in the clinical parameters could be detected within this study population.

\section{Discussion}

Looking at all detected genetic markers we could describe two major types of marker gene combinations represented by group $1 \mathrm{~A}$ and group $2 \mathrm{~B}$. All other groups depict a gradual transition of marker gene combinations between these two groups. Thus the main focus on attention should be on these two groups. Group $1 \mathrm{~A}$ is characterized by the presence of $c j 1365 c, c j 1585 c$, dimeric tlp7 [2], cj1321cj1326, fucP, cj0178, cfrA/cj755, and $c e u E_{11168}$ as well as the absence of $a n s B, d m s A, g g t$ and $c s t I I$. In contrast to that, group $2 \mathrm{~B}$ is an inverted mirror image of this constellation: positive for $a n s B, d m s A, g g t$ but negative for cj1365c, cj1585c, dimeric tlp7 [2], cj1321- cj1326, fucP, cj0178, cfrA/cj755, ceuE $E_{11168}$ as well as cstII/III.

Champion and coworkers identified the flagellin $O$ glycosylation locus cj1321-cj1326 as marker present in livestock-associated strains, whereas $55.7 \%$ of clinical isolates were shown by them to be negative for this gene cluster [6]. According to their data, cj1321-cj1326-negative strains originate mostly from asymptomatic carriers and the environment [6]. Due to our data, $63.9 \%$ of the tested C. jejuni isolates show livestock association based on the presence of $c j 1321-c j 1326$. But in contrast to their findings the non-livestock-associated group $2 \mathrm{~B}$ is significantly more often associated with human origin and thus, bears obviously higher pathogenic potential for humans than the livestock-marker positive strains.

The fucP gene was shown to be present only in isolates negative for $g g t$ [8], which is in accordance with our findings. The ggt-positive group 2 is almost completely free of fuc $P$-positive isolates. Interestingly, group 6 isolates, positive for the $g g t$-associated marker genes ans $B$ and $d m s A$ but not for $g g t$, are mostly able to utilize L-fucose. The fucP distribution pattern is similar to that of the livestockassociation marker genes cj1321-cj1326 and the serine protease Cj1365 [2]. Thus, fucP should be considered as a further marker for livestock association. It can be suggested that the fucose permease is a crucial prerequisite for dwelling in the mucosa layer, while it enables the bacterial cell to metabolize mucosal L-fucose.

The ability to acquire iron is an essential prerequisite for bacterial replication and thus an important virulence factor especially in iron restricted environments $[17,18]$. While C. jejuni has no own siderophores [10] it makes use of exogenous siderophores produced by accompanying bacterial species [19]. At all five different iron uptake systems have been detected in the genome of $C$. jejuni NCTC 11168 [10], but the genome sequence of strain 81-176 
Table 2 Primer

\begin{tabular}{|c|c|c|c|c|}
\hline Gene & Primer name & Sequence $5^{\prime}-3^{\prime}$ & Annealing temp & Reference \\
\hline \multirow[t]{2}{*}{ cj0178 } & cj0178-F01 & TGTAGGCGGGGGTGGCAAGA & $54.0^{\circ} \mathrm{C}$ & this study \\
\hline & cj0178-R01 & ACGACCGCGAGCAGAATTGC & & \\
\hline \multirow[t]{2}{*}{ cj0755/cfrA } & cj0755/cfrA-F01 & ATGGCCGCGAAGTCGTAGGG & $54.0^{\circ} \mathrm{C}$ & this study \\
\hline & cj0755/cfrA-R01 & AGCGATCTATTTGCCACTCGCCT & & \\
\hline \multirow[t]{2}{*}{ cj1321 } & CjNCTC11168-1321_f & AAAATGTCATCATCATAGGAGCG & $60.0^{\circ} \mathrm{C}$ & [6] \\
\hline & CjNCTC11168-1321_r & TCTAAGTTAACGCAAGGCAACA & & \\
\hline \multirow[t]{2}{*}{ cj1322 } & CjNCTC11168-1322_f & GACTTIGGTTTAATGGGTAAGCA & $59.6^{\circ} \mathrm{C}$ & [6] \\
\hline & CjNCTC11168-1322_r & TTCCGGCGTTAAAATTAGAAAA & & \\
\hline \multirow[t]{2}{*}{ cj1323 } & CjNCTC11168-1323_f & AGAACGATTTACCCCATTGAAA & $59.7^{\circ} \mathrm{C}$ & [6] \\
\hline & CjNCTC11168-1323_r & ATTGGTAAAGCTCCTCGATTG & & \\
\hline \multirow[t]{2}{*}{ cj1324 } & CjNCTC11168-1324_f & TGCCGTAAGTGGAGGTAAAGAT & $60.0^{\circ} \mathrm{C}$ & [6] \\
\hline & CjNCTC11168-1324_r & TCTGCACACATTGTTCTATCCC & & \\
\hline \multirow[t]{2}{*}{ cj1325 } & CjNCTC11168-1325_f & ACGGATTACTTITTCCAGATGGT & $60.0^{\circ} \mathrm{C}$ & [6] \\
\hline & CjNCTC11168-1325_r & TTGCTITGAAAATACGCTGAA & & \\
\hline \multirow[t]{2}{*}{ cj1326 } & CjNCTC11168-1326_f & TACATTTCATCGATAAAGCCGA & $59.7^{\circ} \mathrm{C}$ & [6] \\
\hline & CjNCTC11168-1326_r & AAATATAATGGTGTGCCGATCC & & \\
\hline \multirow[t]{4}{*}{ fucP } & cj0486FWD & GATAGAGCATTAAATTGGGATG & $52.0^{\circ} \mathrm{C}$ & {$[8]$} \\
\hline & cj0486REV & CCTATAAAGCCATACCAAGCC & & \\
\hline & rpoC & GAACTTGCTATTGCTGAGCC & & \\
\hline & rpsL & ACCCTAGTGCAAACTCCCCT & & \\
\hline \multirow[t]{3}{*}{ ceuE } & ceuE-81176F01 & GATAGAGTCGCAGGCGTTCC & $60^{\circ} \mathrm{C}$ & this study \\
\hline & ceuE405F & GATAAAGTCGTTGGCGTTCC & & [7] \\
\hline & ceuE405R & GCGAGATTGGAGGACCAAAGG & & \\
\hline \multirow[t]{3}{*}{ pldA } & pldA-81178F01 & AAACTTATGCGTTITT & $45^{\circ} \mathrm{C}$ & this study \\
\hline & pldA-84fwd & AAGCTTATGCGTTITI & & [7] \\
\hline & pld-981rev & TATAAGGCTTTCTCCA & & \\
\hline \multirow[t]{2}{*}{ cstll } & orf7ab & ACTACACTTTAAAACATTTAATCC AAAATCA & $56^{\circ} \mathrm{C}$ & [14] \\
\hline & orf7ab & CCATAAGCCTCACTAGAAGGTATGAGTATA & & \\
\hline \multirow[t]{2}{*}{ cstlll } & orf7c & TTGAAGATAGATATTITGTGGGTAAA & $56^{\circ} \mathrm{C}$ & [14] \\
\hline & orf7c & CTITAAGTAGTGTITATGTCACTTGG & & \\
\hline
\end{tabular}

reveals three fundamental differences in this regard [9]. Cju15, a protein of unknown function, replaces the gene cfrA/cj0755, which encodes a ferric uptake receptor [9]. A second iron uptake transport system encoded by the genes cj0173c-cj0182 is missing critical components e.g. cj0178 and tonB3 [9], and in the gene cluster encoding the enterochelin uptake system cju30 is inserted between cj1355 and cj1356c [9]. Additionally the enterochelin uptake system (CeuBCDE; Cj1352 to Cj1355) is ubiquitous within the $C$. jejuni population, but it shows sequence variability detectable by PCR using different primers. A C. jejuni subpopulation, associated with a higher rate of bloody diarrhea requiring hospitalization, was identified by Feodoroff and coworkers [7]. This subpopulation was positive for $g g t$, but $c e u E$ was not detectable using ceuE-primers derived from the NCTC 11168 genome sequence. This subpopulation corresponds to group 2 in our scheme. In a significant number of group 2 isolates it was only possible to detect the ubiquitous gene for $c e u E$ using primers derived from the genome sequence of C. jejuni strain 81-176 (for pldA we detected no significant differences). In this group of isolates the iron uptake system components cj0178 and $c f r A / c j 755$ are absent in nearly $100 \%$ of the isolates. Thus, the two groups identified by Feodoroff et al. associated with bloody stools/GGT-production and an increased hospitalization rate/ceuE $E_{11168}$-presence overlap to a larger part that corresponds to group 2B. Besides ggt and $c e u E_{11168}, c j 0178$ and $c f r A / c j 755$ should be considered as marker genes correlating with clinical data.

Parker et al. defined, according to the organization of the LOS locus, various LOS locus classes (LLC). The 
LOS locus of LLC A, B, C, M and R includes the sialic acid synthase (neuBCA) and two class-specific sialyltransferases: cstII in LLC A, B, M, R and cstIII in LLC C $[19,20]$. It was demonstrated that the LOS plays a role for epithelial cell invasion [4] and is associated with the clinical course of gastro-enteritis [5]. In this study, we detected just the key-enzymes for LOS sialylization cstII and cstIII. Besides the isolates of the groups $2 \mathrm{~B}$ and 6, the test population was either cstII or cstIII positive. Group $1 \mathrm{~A}$ and $1 \mathrm{~B}^{*}$ isolates were predominantly positive for cstIII. This corresponds to the results of Habib et al. that CC 21 belongs to either LCC C or LCC A [3]. The subgroup $1 \mathrm{~B}^{* *}$, consisting of CC 48 and 206 isolates, is only cstII but not cstIII positive, corresponding mostly to LLC B $[3,15]$. The isolates of the subgroup $1 B^{* * * *}$ (CC 49 and $C C 446)$ were demonstrated to be partially positive, partially negative for cstII but generally cstIII-negative. This corresponds to LLC B and D due to few isolates described by Habib et al. [3]. The majority of group 2A isolates was tested positive for cstII, corresponding to LCC A1 and B $[3,16]$ in contrast to group $2 \mathrm{~B}$ isolates that were tested negative for both cstII and cstIII and belong to LLC D and $\mathrm{E}(\mathrm{H})$ [3]. Positive tested for cstII but not cstIII was the majority of isolates in group 3 . An exclusion were the isolates of CC 353 that are cstIII-positive (corresponding to LCC C). The negative test result for cstII- and cstIII of the majority of isolates in the groups 4, 5, and 6 implies that they belong to LLCs with non-sialylated LOS. Hotter et al. associated LCC D and E, corresponding to group $2 \mathrm{~B}$ in our study, with an increased hospitalization rate [5], that is in accordance with the results obtained by Feodoroff and coworkers for the ggt-positive and $\mathrm{ceuE}_{11168}$-negative group [6] as well as with our prevalence rates for isolates of human origin. In contrast to our data and the data of Feodoroff et al. [7] Hotter and coworkers associated LCC B and C with a higher frequency of bloody stools [5]. This group of isolates corresponds for the most part to the group 1 but also $2 \mathrm{~A}$.

\section{Conclusions}

In general, the arrangement of the eight additional marker genes and the ratio of isolates of human origin substantiates and complements our prior definition of the subgroups.

One outstanding population formed by the groups $1 \mathrm{~A}+\mathrm{B}$, which is able to utilize L-fucose, seems to be livestock-adapted due to the presence of cj1321-cj1326, cj1365c and cstII and/or cstIII, and has all of the five identified putative iron uptake systems of $C$. jejuni. These strains do not exhibit the genes for an extended amino acid metabolism. Due to their livestock adaptation these isolates are less prevalent in humans and secondarily associated with less severe campylobacteriosis.
Contrary to that, group 2 isolates possess an extended amino acid metabolism (positive for ansB, dmsA, as well $g g t$ ) and are not able to metabolize L-fucose (fucP-negative). Group 2 isolates possess only three of five iron uptake systems. This group splits into the two subgroups $2 \mathrm{~A}$ and $2 \mathrm{~B}$. The subgroup $2 \mathrm{~B}$ is additionally negative for the livestock markers cj1365c, cj1321cj1326, as well as cstII/III. In contrast to that, subgroup $2 \mathrm{~A}$ is positive for $c j 1365 c$ and $c s t I I$, but cj1321- cj1326 is likewise not present. Additionally, subgroup 2A is characterized by the presence of the flagellum-secreted nonflagellar protein A1 encoded by $f_{s p A 1}$ [20]. The remaining subgroups demonstrate a somewhat intermediate marker gene profile compared to $1 \mathrm{~A}$ and $2 \mathrm{~B}$. In this respect, group 6 seems noteworthy, as the corresponding isolates are positive for $a n s B$ and $d m s A$, typical for group 2 as well as fucP, cj0178, cj0755 and cj1365c typical for group 1 but not ggt or cj1321- cj1326. Furthermore, only half of group 6 isolates posses a sialylated LOS.

The high virulent isolate subpopulations identified by Mortensen, who associated LCC D and E with a higher hospitalization rate [5] and these of Feodoroff, who associated ggt and a ceuE gene, that is not detectable with primers based on the NCTC 11168 sequence, with severe campylobacteriosis and bloody diarrhea [7], seem to overlap at least partially in group 2 , with the highest pathogenic potential i.e. the highest virulence for humans. Surprisingly, the asymptomatic colonizers identified by Champion et al. [6] and isolates bearing a nonsialylated LOS seem to predominate this high virulent isolate group.

Finally, it should be questioned especially for cstII/III, if there is a causal relationship between a particular genetic marker and clinical parameters, while particular genetic markers are associated with each other and the causal relationship to clinical parameters could be due to a causal relation of an associated genetic marker.

\section{Methods}

\section{C. jejuni isolates}

A total of 266 C. jejuni isolates, 128 of human, 66 of chicken, 45 of bovine, and 27 of turkey origin, with already determined MLS-type and characterized for six genetic markers were selected from our collection [2]. That means about half of the isolates were of human (128) and half of animal (138) origin, what should help to make statements about the clinical relevance of a particular isolate group due to the proportion of isolates originating from human stool samples. The avian and bovine isolates were obtained from the German Campylobacter reference center at the Bundesinstitut für Risikobewertung (Federal Institute for Risk Assessment) in Berlin, Germany. The human isolates originate from stool samples of hospitalized patients of the 
University Medical Center Göttingen, Germany (40\%) as well as outpatients of several doctor's offices in the city of Göttingen (60\%). For these strains the parameters watery diarrhea $(85 \%)$ vs. bloody diarrhea $(15 \%)$ are known. Additionally 42 well-characterized isolates of the CampyNet research network strain collection were included as references. All isolates of this study were PCR-positive for $c i a B$ and the $c d t B$.

The $C$. jejuni isolates were cultured on Columbia agar base (Merck) supplemented with 5\% sheep blood (BA) and incubated at $42^{\circ} \mathrm{C}$ under microaerophilic conditions $\left(5 \% \mathrm{O}_{2}, 10 \% \mathrm{CO}_{2}, 85 \% \mathrm{~N}_{2}\right.$ ) for 24 hours prior to DNA extraction.

\section{DNA extraction and marker gene detection}

Genomic DNA of $C$. jejuni was isolated using the QIAamp DNA Mini Kit (Qiagen) according to the manufacturer's instructions. For detection of the different genetic markers the primers listed in Table 2 were used.

\section{Phylogenetic analysis}

For construction of a UPGMA-dendrogram (unweightedpair group method using average linkages) the MEGA4 software was used [21], and the C. jejuni MLST website (http://pubmlst.org/campylobacter/) developed by Keith Jolley and Man-Suen Chan, sited at the University of Oxford was consulted for assignation of sequence types and clonal complexes [22].

\section{Statistical analyses}

Statistical analysis was performed using the Statistica software. The $\chi^{2}$-test was used to test for significant differences/similarities in the frequencies of the various genetic markers within the defined groups. The obtained $\mathrm{p}$-values are indicated in Table 1.

\section{Abbreviations}

GBS: Guillain-Barré-syndrome; LOS: lipoologosaccharide;

LLC: lipoologosaccharide locus classes; ggt: gamma-glytamyl transpeptidase gene; ceuE: enterochelin uptake binding protein; ciaB: Campylobacter invasion antigen $B$ gene; $c d t B$ : cytolethal distending toxin subunit B gene; cj: gene numbering based on the genome sequence of Campylobacter jejuni strain NCTC 11168; cfrA: ferric receptor gene in C jejuni (iron uptake protein); fucP: L-fucose permease gene; pldA: outer membrane phospholipase A gene; cstII/II: lipooligosaccharide sialyltransferases II and III genes;

ansB: asparaginase gene with an $\mathrm{N}$-terminal sec-dependent secretion signal; $d m s$ : dimethyl sulfoxide oxidoreductase subunit A gene; t/p7: transducer like protein 7 gene; UPGMA: unweighted-pair group method using average linkages; MLST: multi-locus sequence typing; CC: clonal complex.

\section{Competing interests}

All authors declare no competing interests.

\section{Authors' contributions}

AEZ conceived the study idea, performed all mathematical analysis and drafted the manuscript, CO performed bacterial culture, DNA isolation and PCR-analysis, AMT performed DNA isolation and MLST-PCR, RL performed DNA sequencing and assisted in drafting the manuscript. UG participated in the study design and helped drafting the manuscript. All authors read, commented and approved the manuscript.

\section{Acknowledgements}

The authors' work was supported by the Deutsche Forschungsgemeinschaft (DFG GR906/13-1) and the Forschungsförderungsprogramm of the Universitätsmedizin Göttingen (UMG), Germany. This publication was funded by the Open Access support program of the Deutsche Forschungsgemeinschaft and the publication fund of the Georg August Universität Göttingen.

Received: 13 March 2012 Accepted: 2 August 2012

Published: 8 August 2012

\section{References}

1. Zautner AE, Herrmann S, Groß U: Campylobacter jejuni - The search for virulence-associated factors. Arch Lebensmittelhyg 2010, 61:91-101.

2. Zautner AE, Herrmann S, Corso J, Tareen AM, Alter T, Groß U: Epidemiological association of different Campylobacter jejuni groups with metabolism-associated genetic markers. Appl Environ Microbiol 2011, 77:2359-2365

3. Habib I, Louwen R, Uyttendaele M, Houf K, Vandenberg O, Nieuwenhuis EE, Miller WG, van Belkum A, De Zutter L: Correlation between genotypic diversity, lipooligosaccharide gene locus class variation, and caco-2 cell invasion potential of Campylobacter jejuni isolates from chicken meat and humans: contribution to virulotyping. Appl Environ Microbiol 2009, 75:4277-4288

4. Louwen R, Heikema A, van Belkum A, Ott A, Gilbert M, Ang W, Endtz HP, Bergman MP, Nieuwenhuis EE: The sialylated lipooligosaccharide outer core in Campylobacter jejuni is an important determinant for epithelial cell invasion. Infect Immun 2008, 76:4431-4438.

5. Mortensen NP, Kuijf ML, Ang CW, Schiellerup P, Krogfelt KA, Jacobs BC, van Belkum A, Endtz HP, Bergman MP: Sialylation of Campylobacter jejuni lipooligosaccharides is associated with severe gastro-enteritis and reactive arthritis. Microbes Infect 2009, 11:988-994.

6. Champion OL, Gaunt MW, Gundogdu O, Elmi A, Witney AA, Hinds J, Dorrell $\mathrm{N}$, Wren BW: Comparative phylogenomics of the food-borne pathogen Campylobacter jejuni reveals genetic markers predictive of infection source. Proc Natl Acad Sci U S A 2005, 102:16043-16048.

7. Feodoroff $B$, Ellström $P$, Hyytiäinen $H$, Sarna S, Hänninen $M L$, Rautelin $H$ : Campylobacter jejuni isolates in Finnish patients differ according to the origin of infection. Gut Pathog 2010, 2:22.

8. Muraoka WT, Zhang Q: Phenotypic and genotypic evidence for L-fucose utilization by Campylobacter jejuni. J Bacterio/ 2011, 193:1065-1075.

9. Hofreuter D, Novik V, Galán JE: Metabolic diversity in Campylobacter jejuni enhances specific tissue colonization. Cell Host Microbe 2008, 4:425-433.

10. Parkhill J, Wren BW, Mungall K, Ketley JM, Churcher C, Basham D, Chillingworth T, Davies RM, Feltwell T, Holroyd S, Jagels K, Karlyshev AV, Moule S, Pallen MJ, Penn CW, Quail MA, Rajandream MA, Rutherford KM, van Vliet AH, Whitehead S, Barrell BG: The genome sequence of the foodborne pathogen Campylobacter jejuni reveals hypervariable sequences. Nature 2000, 403:665-668.

11. Richardson PT, Park SF: Enterochelin acquisition in Campylobacter coli: characterization of components of a binding-protein-dependent transport system. Microbiology 1995, 141:3181-3191.

12. Grant KA, Belandia IU, Dekker N, Richardson PT, Park SF: Molecular characterization of pldA, the structural gene for a phospholipase A from Campylobacter coli, and its contribution to cell-associated hemolysis. Infect Immun 1997, 65:1172-1180.

13. Parker CT, Gilbert M, Yuki N, Endtz HP, Mandrell RE: Characterization of lipooligosaccharide-biosynthetic loci of Campylobacter jejuni reveals new lipooligosaccharide classes: evidence of mosaic organizations. J Bacteriol 2008, 190:5681-5689.

14. Parker CT, Horn ST, Gilbert M, Miller WG, Woodward DL, Mandrell RE: Comparison of Campylobacter jejuni lipooligosaccharide biosynthesis loci from a variety of sources. J Clin Microbiol 2005, 43:2771-2781.

15. Hotter GS, Li IH, French NP: Binary genomotyping using lipooligosaccharide biosynthesis genes distinguishes between Campylobacter jejuni isolates within poultry-associated multilocus sequence types. Epidemiol Infect 2010, 138:992-1003.

16. Revez J, Rossi M, Ellström P, de Haan C, Rautelin H, Hänninen ML: Finnish Campylobacter jejuni Strains of Multilocus Sequence Type ST-22 Complex Have Two Lineages with Different Characteristics. PLoS One 2011, 6:e26880. 
17. Pickett CL, Auffenberg T, Pesci EC, Sheen VL, Jusuf SS: Iron acquisition and hemolysin production by Campylobacter jejuni. Infect Immun 1992, 60:3872-3877.

18. van Vliet AH, Ketley JM, Park SF, Penn CW: The role of iron in Campylobacter gene regulation, metabolism and oxidative stress defense. FEMS Microbiol Rev 2002, 26:173-186.

19. Field LH, Headley VL, Payne SM, Berry LJ: Influence of iron on growth, morphology, outer membrane protein composition, and synthesis of siderophores in Campylobacter jejuni. Infect Immun 1986, 54:126-132.

20. de Haan CP, Kivistö R, Hänninen ML: Association of Campylobacter jejuni Cj0859c gene ( $f s p A)$ variants with different $C$. jejuni multilocus sequence types. Appl Environ Microbiol 2010, 76:6942-6943.

21. Kumar S, Nei M, Dudley J, Tamura K: MEGA: a biologistcentric Software for evolutionary analysis of DNA and protein sequences. Brief Bioinform 2008, 9:299-306.

22. Jolley KA, Chan MS, Maiden MC: mlstdbNet-distributed multi-locus sequence typing (MLST) databases. BMC Bioinformatics 2004, 5:86.

doi:10.1186/1471-2180-12-171

Cite this article as: Zautner et al: Epidemiological association of Campylobacter jejuni groups with pathogenicity-associated genetic markers. BMC Microbiology 2012 12:171.

\section{Submit your next manuscript to BioMed Central and take full advantage of:}

- Convenient online submission

- Thorough peer review

- No space constraints or color figure charges

- Immediate publication on acceptance

- Inclusion in PubMed, CAS, Scopus and Google Scholar

- Research which is freely available for redistribution 